\title{
Osteoblastoma of the Lateral Skull Base: Work-Up, Surgical Management, and a Review of the Literature"
}

\author{
Craig Miller $^{1}$ Rihan Khan ${ }^{2}$ \\ G. Michael Lemole, Jr. ${ }^{3}$ \\ ${ }^{1}$ Division of Otolaryngology, The University of Arizona Ear Institute, \\ University of Arizona, Arizona, USA \\ 2 Department of Radiology, The University of Arizona, Arizona, USA \\ ${ }^{3}$ Division of Neurosurgery, The University of Arizona, Arizona, USA
}

Abraham Jacob ${ }^{1}$

\begin{abstract}
Address for correspondence Abraham Jacob, MD, Arizona Cancer Center; 1515 N. Campbell Ave, Room 0973, Tucson, AZ 85719, USA (e-mail: ajacob@surgery.arizona.edu).
\end{abstract}

J Neurol Surg Rep 2013;74:37-42.

\section{Introduction}

Osteoblastomas are rare bone tumors, accounting for approximately $1 \%$ of all primary bone neoplasms. These tumors
Objectives To describe the work-up and surgical management of an osteoblastoma involving the lateral skull base. Typically occurring in the spine or long bones, osteoblastomas of the craniofacial skeleton are exceedingly rare and infrequently reported. A review of the current literature regarding temporal bone osteoblastoma, diagnosis, and treatment is described.

Methods This case report describes the clinical presentation, radiographic studies, surgical management, histology, and postoperative follow-up of a young man presenting to a tertiary care neurotology practice with osteoblastoma involving the lateral skull base. A review of the current literature regarding osteoblastoma of the skull base, workup, and treatment is described.

Results A 15-year-old adolescent boy with a greater than 1-year history of right-sided retroauricular pain, a palpable postauricular mass, and chronic headaches presented for evaluation/management. Microscope examination of the ears, hearing, and cranial nerve function were normal. High-resolution temporal bone computed tomography and magnetic resonance imaging scans were obtained, which revealed an expansile mass involving the junction of the temporal and occipital bones. The patient underwent a combined retrosigmoid/retrolabyrinthine resection of this extradural tumor. Histology revealed a benign bone neoplasm consistent with osteoblastoma. Complete surgical resection was achieved, and the patient's symptoms fully resolved. Follow-up imaging studies found no evidence of recurrence. The scientific literature relevant to work-up and management of osteoblastoma is reviewed.

Discussion Osteoblastomas of the lateral skull base are rare, histologically benign tumors that can present with radiographic features suggestive of malignancy. An en bloc resection is important for both diagnosis and definitive treatment of these neoplasms. The differential diagnosis on imaging and histology is discussed. received

December 24, 2012 accepted after revision

February 25, 2013

published online

June 13, 2013 typically arise in the spine and show a 3:1 male predominance. ${ }^{1}$ Lesions involving the temporal bone are extremely rare, with 22 total cases reported in the English-language literature and only 8 cases reported in otolaryngology over (c) 2013 Georg Thieme Verlag KG Stuttgart · New York
DOI http://dx.doi.org/ $10.1055 / \mathrm{s}-0033-1346978$. ISSN 2193-6358. 

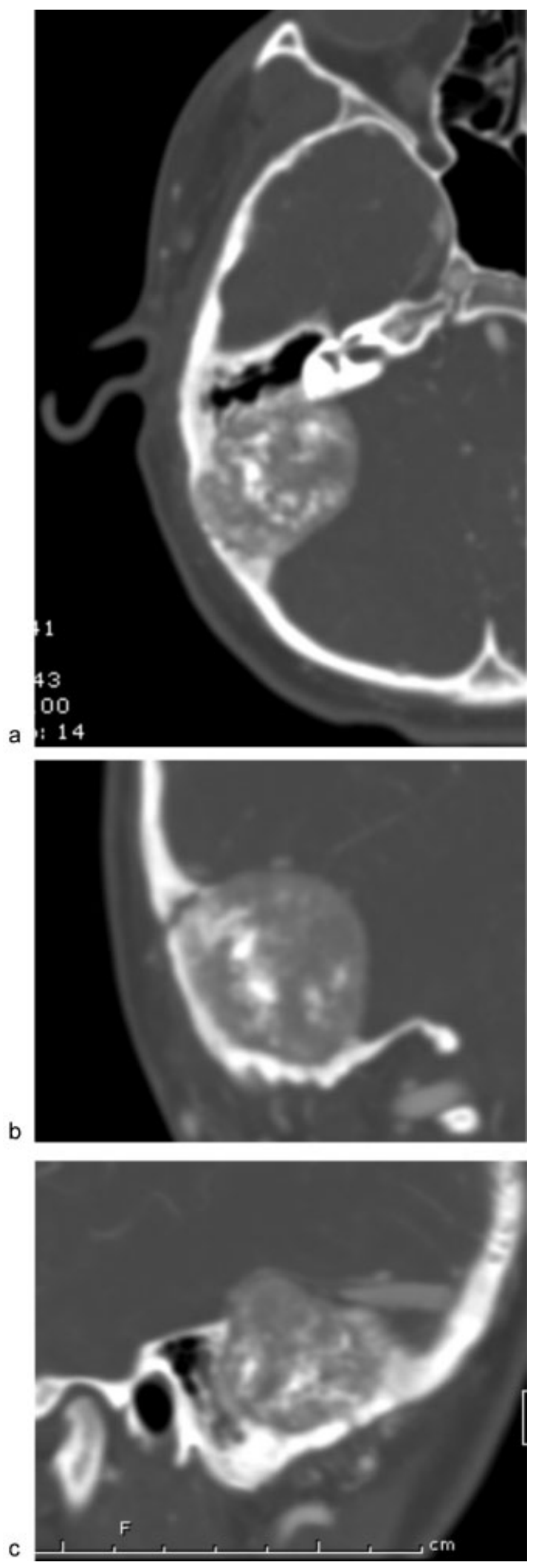

Fig. 1 (a) Axial contrast-enhanced computed tomography (CT) and (b) coronal computed tomography angiography (CTA) images show a large heterogeneous mass along the posterior aspect of the right temporal bone with dense calcifications. (c) Sagittal CTA image shows that the transverse sinus tapers to a point (arrow) at the superior margin of the lesion with no definite continuation. the past 20 years. $^{2}$ Of the reported cases, complete resection of tumor has only been attempted a handful of times, and a combined retrosigmoid, retrolabyrinthine approach has not previously been described to our knowledge.

\section{Observation}

An otherwise healthy 15-year-old boy presented for evaluation of right-sided postauricular pain. The pain was localized, and there were no associated hypoacusis, tinnitus, otorrhea, dizziness, or focal neurologic deficits. The patient was tender behind the right ear, with boggy edema of the skin overlying the bone. An audiogram was normal.

Initially, the patient's primary care physician prescribed pain medications for management; however, due to persistence of symptoms, imaging was ultimately obtained. The patient underwent a contrast-enhanced computed tomography (CT) scan (-Fig. 1a), which showed a heterogeneous, enhancing 4-cm mass lesion abutting the posterior aspect of the right temporal bone. The lesion was expansile, thinning overlying posterior fossa calvarium and invading the posterior aspect of the mastoid air cells. Many small internal calcifications were also noted. Subsequent CT angiography of the head ( $\mathbf{- F i g}$. 1b, c) demonstrated that the right transverse sinus was severely tapered to its transverse/sigmoid junction.

Magnetic resonance imaging (MRI)/magnetic resonance venography (MRV) of the head showed that the majority of the mass avidly enhanced with several small internal foci that did not ( $\mathbf{- F i g . ~ 2 a , ~ b ) . ~ T h e ~ l e s i o n ~ a p p e a r e d ~ t o ~ b e ~ w e l l ~ e n c a p - ~}$ sulated and completely extradural. There was no evidence on any imaging modality to suggest that the lesion had intradural involvement. The lesion had no T2 signal within it (-Fig. 2c), and it also demonstrated a large amount of dark signal on the gradient images (-Fig. 2d); this indicated a relative lack of water and a large amount of calcification within the lesion, respectively. MRV also showed severe tapering and probable occlusion at the right transverse/ sigmoid sinus junction. Differential diagnosis at this time included benign bone processes, such as osteoid osteomas, aneurysmal bone cysts, or fibrous dysplasia, and malignant processes, such as osteogenic sarcomas, chondrosarcoma, or metastatic disease. Even with extensive imaging, a diagnosis was not achieved. Though the heterogeneous nature of the mass directed our decision making toward a bony process. The extradural nature of the mass made surgical en bloc resection an appropriate treatment option.

Thus, due to the large size of the tumor, the patient's age, and the fact that the differential diagnosis included malignancy, surgical management was offered for both diagnostic and curative purposes. The patient underwent a combined retrolabyrinthine/retrosigmoid dissection via a right transtemporal approach. Neuronavigation (BrainLab; Westchester, Illinois, USA) and neuromonitoring (cranial nerves V and VII as well as somatosensory evoked potentials [SSEPs] and cortical evoked potentials) were utilized intraoperatively.

Initially, a limited retrosigmoid craniotomy was performed to allow posterior exposure of the tumor and control of the 

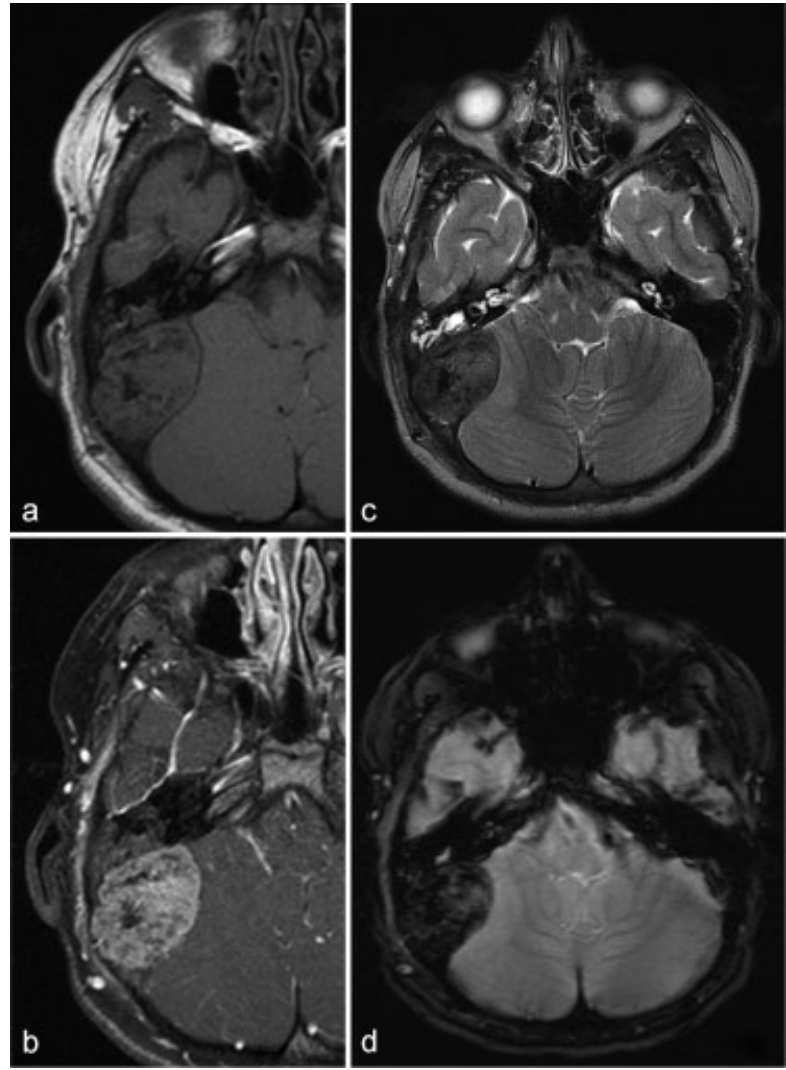

Fig. 2 (a) Axial T1 precontrast and (b) T1 postcontrast magnetic resonance imaging (MRI) images show a T1 dark mass lesion, which avidly enhances. (c) Axial T2 MRI shows that the lesion is significantly dark, indicating the relative lack of water content in the mass, whereas (d) axial gradient MRI shows extensive dark signal related to the calcification.

transverse sinus. This was followed by a mastoidectomy to allow for full exposure of the tumor mass that involved a significant portion of the posterior temporal bone. The mass extended from the tegmen all the way into the retrofacial air cell system, as well as a significant portion of the posterior petrous bone from tegmen to retrofacial air cells. Important landmarks were identified, including the tegmen, the region of the sinodural angle, and the ear canal, incus, horizontal semicircular canal, and the vertical segment of the facial nerve. The bone surrounding these structures was noted to be soft, doughy, and highly vascularized. Biopsies were taken and sent for frozen section and permanent section analysis. Intraoperative pathology revealed a bone-forming neoplasm with immature bone as well as spindle cell populations with rich vascularity ( - Fig. $\mathbf{3}$ ). The pathology team read the sample as a "benign tumor, otherwise unspecified." There were no malignant features noted such as increased nuclear to cytoplasmic ratio or extensive mitotic figures to suggest a malignant process. At this point it was felt that a total en bloc resection would be curative, and the surgeons proceeded with the operation.

Large diamond burrs were used to progressively remove the tumor mass down to normal dura and normal surrounding bone. On initial inspection, the otic capsule appeared to be

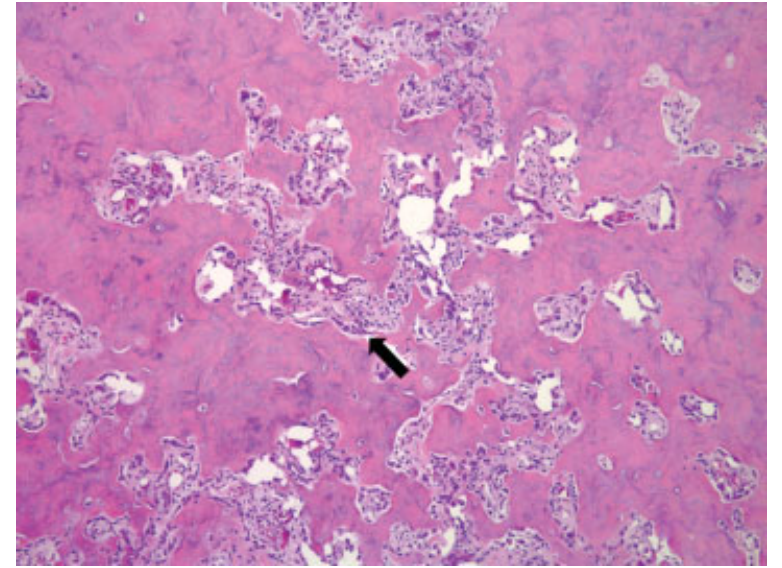

Fig. 3 Hematoxylin and eosin (H\&E) stained slide at $200 \times$ magnification shows a bone-forming neoplasm with immature bone (arrow) and spindle cell population with rich vasculature.

spared. Following the retrolabyrinthine dissection, a Spetzler curette was used to completely elevate the tumor off normalappearing, intact dura in the middle fossa. The facial nerve was then fully delineated and preserved, from external genu to the stylomastoid foramen. The bone surrounding the facial nerve and the retrofacial system was relatively normal and therefore felt to be free of tumor burden.

The only area of questions as to the integrity of the otic capsule was at the posterior semicircular canal. The operculum was identified, as was the transition from the endolymphatic sac to the endolymphatic duct. At the endolymphatic duct, the bone remained quite soft until the otic capsule, which appeared to be more resistant to bone destruction, and thus it was felt that this portion was free of tumor burden.

At this point, there appeared to be normal, intact dura in the middle fossa, the sinodural angle region, and down onto the sigmoid sinus. There was some initial bleeding from the sigmoid sinus that was easily controlled using Gelfoam (Pfizer, New York, New York, USA) and Surgicel (Ethicon, Somerville, New Jersey, USA). The wound was adequately irrigated and it was felt that gross resection of the tumor was achieved. The aditus ad antrum was closed off with a large piece of thin, implantable AlloDerm (LifeCell, Bridgewater, New Jersey, USA) to prevent a potential cerebrospinal fluid (CSF) leak, though the tumor was entirely extradural and there was no leak noted intraoperatively. Next, large pieces of Gelfoam were placed in the bony defect and a cranioplasty was performed using the patient's native retrosigmoid craniotomy bone flap, secured with an OsteoMed (Addison, Texas, USA) titanium cranioplasty plate. The drilled out mass was then packed with Gelfoam and a piece of titanium mesh was placed over the mastoid to recontour the normal surface area of the bone. Next, the muscle and galea were reapproximated together and the deep dermal layer was reapproximated with 3-0 Vicryl suture. The dermis was then reapproximated with an external continuous locking stitch using 3-0 nylon suture. A compression ear dressing was placed, and the patient was returned to anesthesiology for extubation and transport to recovery. 

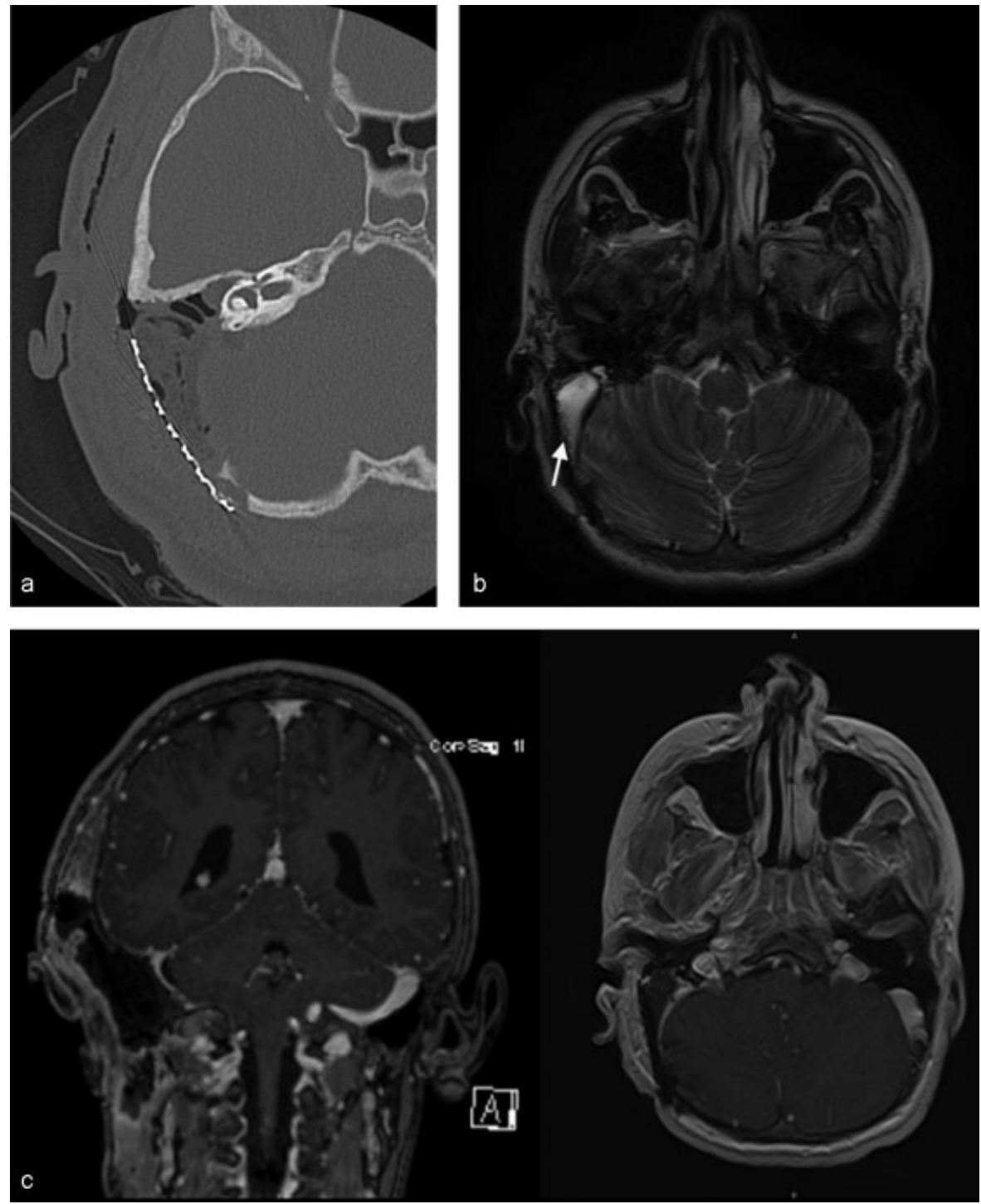

Fig. 4 (a) Immediate postoperative axial noncontrast computed tomography (CT) image shows the large postoperative skull defect with graft and air. No residual tumor was seen on CT or magnetic resonance imaging (MRI). (b) Postoperative T2 bright fluid in the resection site. No evidence of residual tumor postoperatively. Note overlying mesh lateral to the operative site (arrow). Coronal and axial magnetic resonance venography (MRV) images show narrowing but patency of the right sigmoid sinus, which severely tapers just before the jugular bulb. (c) The jugular bulb was widely patent.

Pathologic analysis of permanent specimens identified osteoblastoma, a benign, bone-forming tumor ( $\mathbf{F i g . ~ 3 ) . ~ T o ~}$ date, this patient has had 12 months of follow-up with no evidence of tumor recurrence. Postoperative temporal bone CT ( - Fig. 4a) and MRI scans are shown (-Fig. 4b). Postoperative MRV again demonstrates probable occlusion of the right sigmoid sinus (-Fig. 4c). The patient has no cranial nerve deficits, headaches/postauricular pain have resolved, and hearing is normal. Evaluation by the medical oncology team found no need for adjuvant therapies such as radiation or chemotherapy.

\section{Discussion}

Osteoblastomas were described, almost concurrently, by Lichenstein and Jaffe in $1956 .{ }^{3,4}$ They are rare, histologically benign, vascular, osteoid-forming neoplasms that account for only $1 \%$ of primary bone tumors. ${ }^{5,6}$ The most frequents sites of involvement are the posterior elements of the vertebrae and metaphyses of long bones; however, up to $15 \%$ arise within the bones of the craniofacial skeleton. ${ }^{7}$ The most common site of involvement in this anatomic location is the maxilla, but other areas, notably the temporal bone, do occur (- Table $\mathbf{1}$ ).

These tumors occur predominantly in young men, with a 3:1 predominance over females. They are usually diagnosed in the second through fourth decades of life. Tumors express high levels of osteoblastic lineage regulatory genes such as Osterix and Runx2. Overexpression of these genes in transgenic mice results in defective osteoblastic maturation with generalized immature bone and an osteopenic phenotype. ${ }^{8}$ Clinically, symptoms are usually nonspecific and include 
Table 1 Twenty-two Reported Cases of Osteoblastomas Located in the Temporal Bone

\begin{tabular}{|c|c|c|c|c|c|c|c|c|}
\hline Author & Year & Age & Sex & Symptoms & Location & Intervention & Length of $\mathrm{F} / \mathrm{U}$ & Outcome \\
\hline Lichtenstein $^{3}$ & 1956 & $9 y$ & $\mathrm{~F}$ & Not described & Rt; $S$ & PR & $13 \mathrm{mo}$ & NR \\
\hline Lichtenstein and Sawyer $^{13}$ & 1964 & $78 \mathrm{y}$ & M & $\mathrm{HA}$ & $\mathrm{U} / \mathrm{K}$ & PR & $4 y$ & NR \\
\hline Ronis et al ${ }^{14}$ & 1974 & $13 \mathrm{y}$ & $M$ & HL, pulsating & Rt & PR & $13 \mathrm{mo}$ & NR \\
\hline Glasscock et al ${ }^{15}$ & 1978 & $57 \mathrm{y}$ & $\mathrm{F}$ & Severe pain & Rt & TR & $\mathrm{U} / \mathrm{K}$ & NR \\
\hline Potter et al ${ }^{16}$ & 1983 & $19 \mathrm{y}$ & $\mathrm{F}$ & OM & $R ; M, P, S$ & $E, P R$ & $18 \mathrm{mo}$ & NR \\
\hline Naclerio et $\mathrm{al}^{17}$ & 1985 & $4 y$ & $\mathrm{~F}$ & $\mathrm{FP}, \mathrm{HL}$ & L; M,P & TR & $12 \mathrm{mo}$ & NR \\
\hline Gellad et al ${ }^{18}$ & 1985 & $30 \mathrm{y}$ & $\mathrm{F}$ & T; puffiness; HL & R; M & PR & $7 y$ & $R$ \\
\hline Miyazaki et al ${ }^{19}$ & 1987 & $7 \mathrm{~m}$ & $\mathrm{~F}$ & Swelling & L; S & TR & $19 \mathrm{mo}$ & NR \\
\hline Matsumoto et a $\left.\right|^{20}$ & 1989 & $29 y$ & $M$ & $\mathrm{HA}$ & $R ; M, P, S$ & TR & $9 \mathrm{mo}$ & NR \\
\hline Adler et $\mathrm{al}^{1}$ & 1990 & $28 \mathrm{y}$ & $\mathrm{F}$ & Pain; FP & $R ; M, P, S$ & $\mathrm{PR}+\mathrm{rad}$ & $5 y$ & $R$ \\
\hline Singer and Deutsch ${ }^{21}$ & 1993 & $16 \mathrm{y}$ & $M$ & Pain & L; M,P & $\mathrm{PR}+\mathrm{rad}$ & $10 y$ & $R$ \\
\hline Khashaba et al ${ }^{22}$ & 1995 & $16 \mathrm{y}$ & $\mathrm{F}$ & Pain; FP & L; M & TR & none & $\mathrm{U} / \mathrm{K}$ \\
\hline Tsuchida and Nagao 23 & 1995 & $27 \mathrm{y}$ & $\mathrm{F}$ & Pain & $R ; S, P$ & TR & $\mathrm{U} / \mathrm{K}$ & $\mathrm{U} / \mathrm{K}$ \\
\hline Ohkawa et al ${ }^{24}$ & 1997 & $28 \mathrm{y}$ & $\mathrm{F}$ & Pain & L; S,P & $E, T R$ & $3 y$ & NR \\
\hline Figueiredo et al ${ }^{25}$ & 1998 & $23 y$ & $M$ & $\begin{array}{l}\text { HL, swelling } \\
\text { and pain }\end{array}$ & L; M,P,S & $E, T R$ & $18 \mathrm{mo}$ & NR \\
\hline Low et $\mathrm{al}^{26}$ & 2000 & $8 y$ & $\mathrm{~F}$ & Swelling & $L ; S$ & TR & $13 \mathrm{mo}$ & NR \\
\hline Doshi et al ${ }^{27}$ & 2001 & $19 y$ & $\mathrm{~F}$ & $\mathrm{~T}, \mathrm{HL}$ & $R ; M, P$ & PR & $15 \mathrm{mo}$ & NR \\
\hline Ugur et al ${ }^{28}$ & 2005 & $45 \mathrm{y}$ & $\mathrm{F}$ & Diplopia and HA & $R ; P$ & PR & $18 \mathrm{y}$ & NR \\
\hline Shimizu et al ${ }^{5}$ & 2006 & $61 \mathrm{y}$ & $\mathrm{F}$ & $\mathrm{HL}$ & L; M,P,S & $E, P R$ & $24 \mathrm{mo}$ & NR \\
\hline Tugcu et a $\left.\right|^{29}$ & 2008 & $23 y$ & $M$ & $\begin{array}{l}\text { Swelling and } \\
\text { tenderness }\end{array}$ & $R$ & $\mathrm{U} / \mathrm{K}$ & $\mathrm{U} / \mathrm{K}$ & $\mathrm{U} / \mathrm{K}$ \\
\hline Pérez-Mora et al ${ }^{9}$ & 2009 & $26 \mathrm{y}$ & $\mathrm{F}$ & $\mathrm{T}, \mathrm{HL}$ & R; M & TR & $10 \mathrm{mo}$ & NR \\
\hline Njim et $\left.a\right|^{2}$ & 2010 & $22 y$ & $\mathrm{~F}$ & Pain, swelling & L; M & TR & $1 \mathrm{y}$ & NR \\
\hline Present case & 2012 & $15 \mathrm{y}$ & $M$ & $\begin{array}{l}\text { Swelling, } \\
\text { tenderness }\end{array}$ & $R ; M, P, S$ & TR & $8 \mathrm{mo}$ & NR \\
\hline
\end{tabular}

Abbreviations: E, embolization; FP, facial palsy; HA, headache; HL, hearing loss; M, mastoid portion; NR, no recurrence; OM, otitis media; P, petrous portion; PR, partial removal; R, recurrence; rad, radiation; Rt, right; S, squamous portion; T, tinnitus; TR, total removal; U/K, unknown/not described.

localized, insidious pain that is unresponsive to anti-inflammatory agents. ${ }^{9}$ There may also be soft tissue swelling and erythema over the area of tumor growth. When the tumor affects the temporal bone and middle ear structures, it can lead to progressive hearing loss (typically conductive) and facial nerve palsy due to compression. ${ }^{9}$

Twenty-two temporal bone osteoblastomas have been described in the literature previously. These patients were treated with either partial resection or total resection. Only 3 of the 22 patients showed recurrence of the tumor. Notably, these three patients only underwent partial tumor removal, and recurrence was likely due to tumor bulk remaining postoperatively.

The radiographic appearance of osteoblastomas is variable and often nonspecific. ${ }^{7}$ On imaging, aggressive osteoblastomas can mimic other tumors, including malignant varieties. The differential diagnosis in such instances consists of osteoid osteomas, aneurysmal bone cysts, osteogenic sarcomas, fibrous dysplasia, chondrosarcoma, and metastatic disease.
Osteoblastomas typically demonstrate intratumoral ossification, including some with a central nidus of sclerotic bone encompassed by a radiolucent halo similar to osteoid osteoma. ${ }^{7}$ Most osteoblastomas remain confined to bone and do not destroy or penetrate cortex; however, up to $12 \%$ have features that mimic a malignant process. ${ }^{10}$

Histologically, osteoblastomas display a predominance of immature bone with high levels of osteoblastic activity. Many are composed of interanastomosing trabeculae of woven bone set within large spindle cell populations with rich vascularity. $^{7}$

Current literature regarding osteoblastoma of the spine suggests that en bloc resection is sufficient as definitive treatment for these tumors. Radiotherapy does not alter the course of the disease and may be contraindicated for fear of malignant degeneration. ${ }^{11}$ We are unaware of any English-language studies specifically evaluating the role of radiation therapy for osteoblastomas of the temporal bone or skull. Additionally, current literature finds no role for 
chemotherapy in primary, singular, benign bone tumors. ${ }^{12}$ Accordingly, after en bloc resection of the tumor, our patient did not undergo any further therapy.

In summary, osteoblastomas are benign bone-forming tumors that rarely involve the craniofacial skeleton. The majority of these neoplasms occur in the spine and long bones. En bloc resection is sufficient and curative, with no adjuvant chemotherapy or radiation necessary.

\section{Acknowledgments}

The authors would like to thank Dr. Achyut Bhattacharyya for his contribution to this manuscript.

\section{Note}

Research supported by the Medical Student Research Program, The University of Arizona College of Medicine, National Institute of deafness and Other communication disorders [AJ - k08 dc009644- 01a1].

\section{References}

1 Adler M, Hnatuk L, Mock D, Freeman JL. Aggressive osteoblastoma of the temporal bone: a case report. J Otolaryngol 1990;19(5): 307-310

2 Njim L, Dhouibi A, Touil N, et al. Benign osteoblastoma in an unusual mastoid location. Eur Ann Otorhinolaryngol Head Neck Dis 2010;127(5):183-185

3 Lichtenstein L. Benign osteoblastoma; a category of osteoid-and bone-forming tumors other than classical osteoid osteoma, which may be mistaken for giant-cell tumor or osteogenic sarcoma. Cancer 1956;9(5):1044-1052

4 Jaffe HL. Benign osteoblastoma. Bull Hosp Jt Dis 1956;17(2): 141-151

5 Shimizu N, Sakata K, Yamamoto I. Benign osteoblastoma of the temporal bone: Case report and review of the literature. Surg Neurol 2006;66(5):534-538

6 Nielsen GP, Rosenberg AE. Update on bone forming tumors of the head and neck. Head Neck Pathol 2007;1(1):87-93

7 Lucas DR. Osteoblastoma. Arch Pathol Lab Med 2010;134(10): 1460-1466

8 Dancer JY, Henry SP, Bondaruk J, et al. Expression of master regulatory genes controlling skeletal development in benign cartilage and bone forming tumors. Hum Pathol 2010;41(12):1788-1793

9 Pérez-Mora RM, Sanchez-Carrion S, Barea FL, Garcia-Raya P, Lassaletta L. Osteoblastoma of the temporal bone. Skull Base 2009;19(5):345-348
10 Lucas DR, Unni KK, McLeod RA, O'Connor MI, Sim FH. Osteoblastoma: clinicopathologic study of 306 cases. Hum Pathol 1994; 25(2):117-134

11 Boriani S, Amendola L, Bandiera S, et al. Staging and treatment of osteoblastoma in the mobile spine: a review of 51 cases. Eur Spine J 2012;21(10):2003-2010

12 Mavrogenis AF, Guerra G, Romantini M, Romagnoli C, Casadei R, Ruggieri P. Tumours of the atlas and axis: a 37-year experience with diagnosis and management. Radiol Med (Torino) 2012; 117(4):616-635

13 Lichtenstein L, Sawyer WR. Benign Osteoblastoma. Further Observations and Report of Twenty Additional Cases. J Bone Joint Surg Am 1964;46:755-765

14 Ronis ML, Obando M, Bucko MI, Liebman EP. Benign osteoblastoma of the temporal bone. Laryngoscope 1974;84(5):857-862

15 Glasscock ME III, Miller GW, Drake FD, Kanok MM. Surgery of the skull base. Laryngoscope 1978;88(6):905-923

16 Potter C, Conner GH, Sharkey FE. Benign osteoblastoma of the temporal bone. Am J Otol 1983;4(4):318-322

17 Naclerio RM, Jenkins HA, Herzog JA. Osteoblastoma of the temporal bone presenting as facial paralysis. Int J Pediatr Otorhinolaryngol 1985;8(3):253-262

18 Gellad FE, Hafiz MA, Blanchard CL. Osteoblastoma of the temporal bone: CT findings. J Comput Assist Tomogr 1985;9(3): 577-579

19 Miyazaki S, Tsubokawa T, Katayama Y, Kai Y, Sakurai I. Benign osteoblastoma of the temporal bone of an infant. Surg Neurol 1987;27(3):277-283

20 Matsumoto K, Kakita K, Fukuma S. Large, benign osteoblastoma of the temporal bone-case report. Neurol Med Chir (Tokyo) 1989; 29(5):444-448

21 Singer JM, Deutsch GP. The successful use of radiotherapy for osteoblastoma. Clin Oncol (R Coll Radiol) 1993;5(2):124-125

22 Khashaba A, De Donato G, Vassallo G, et al. Benign osteoblastoma of the mastoid part of the temporal bone: case report. J Laryngol Otol 1995;109(6):565-568

23 Tsuchida T, Nagao S. Benign osteoblastoma of the temporal bone: MRI. Neuroradiology 1995;37(4):326-327

24 Ohkawa M, Fujiwara N, Tanabe M, et al. Benign osteoblastoma of the temporal bone. AJNR Am J Neuroradiol 1997;18(2):324-326

25 Figueiredo EG, Vellutini E, Velasco O, Siqueira M, Bougar P. Giant osteoblastoma of temporal bone. Case report. Arq Neuropsiquiatr 1998;56(2):292-295

26 Low Y, Foo CL, Seow WT. Childhood temporal bone osteoblastoma: a case report. J Pediatr Surg 2000;35(7):1127-1129

27 Doshi SV, Frantz TD, Korol HW. Benign osteoblastoma of the temporal bone: case report and literature review. Am J Otolaryngol 2001;22(3):211-214

28 Ugur HC, Torun F, Kanpolat Y. Petrous bone osteoblastoma invading the cavernous sinus. J Clin Neurosci 2005;12(4):489-492

29 Tugcu B, Gunaldi O, Gunes M, Tanriverdi O, Bilgic B. Osteoblastoma of the temporal bone: a case report. Minim Invasive Neurosurg 2008;51(5):310-312 